\title{
Three-dimensional reconstruction of corpora allata nucleus reveals insights into epigenetic mechanisms of caste differentiation in Melipona scutellaris stingless bees
}

\author{
N. A. B. Schumann ${ }^{1}$, C. A. M. Cardoso-Junior ${ }^{2}$, E. H. Silva ${ }^{3}$, C. Ueira-Vieira ${ }^{1}$, \\ M. E. Beletti ${ }^{4}$, M. B. Franco ${ }^{4}$, M. Bezerra-Laure ${ }^{5}$, G. R. Araujo ${ }^{1}$, \\ B. A. N. Travençolo ${ }^{3}$, A. M. Bonetti ${ }^{1}$ \\ ${ }^{1}$ Instituto de Biotecnologia, Universidade Federal de Uberlândia, Uberlândia, MG 38400-902, Brazil \\ ${ }^{2}$ Departamento de Biologia Celular, Molecular e Bioagentes Patogênicos, Faculdade de Medicina de Ribeirão Preto, \\ Universidade de São Paulo, Ribeirão Preto, SP 14049-900, Brazil \\ ${ }^{3}$ Faculdade de Computação, Universidade Federal de Uberlândia, Uberlândia, MG 38400-902, Brazil \\ ${ }^{4}$ Instituto de Ciências Biomédicas, Departamento de Morfologia, Universidade Federal de Uberlândia, Uberlândia, MG \\ 38400-902, Brazil \\ ${ }^{5}$ Departamento de Genética, Faculdade de Medicina de Ribeirão Preto, Universidade de São Paulo, Ribeirão Preto, SP \\ 14049-900, Brazil
}

Received 9 October 2018 - Revised 19 February 2019 - Accepted 19 March 2019

\begin{abstract}
In most Hymenoptera, the caste differentiation is influenced by differential environmental cues (e.g., food), which affect larvae development. However, Melipona stingless bees represent an exception to this rule. Workers and queens can emerge from brood cells of the same size, with the same quantity and quality of food. The different phenotypes of female bees depend on the interaction of genetic and environmental components. This mechanism is driven by the juvenile hormone, a key physiological factor synthetized by the corpora allata glands. In addition to that, epigenetic mechanisms were recently associated with caste development in Melipona scutellaris. In this study, using images captured by confocal microscopy and analyzed by 3D computational reconstruction, we show the similarities and differences in the pattern and volume of heterochromatin in the cell nuclei of corpora allata glands of $M$. scutellaris . Our results imply that heterochromatin territorial dispersion may act as a major player on the female phenotypic plasticity of $M$. scutellaris stingless bees.
\end{abstract}

DNA methylation / chromatin / YO-PRO-1-iodice / histone post-translational modifications / epigenetics

\section{INTRODUCTION}

In social insects, the females are divided into two castes: queens (fertile females) and workers

Electronic supplementary material The online version of this article (https://doi.org/10.1007/s13592-019-00647-2) contains supplementary material, which is available to authorized users.

Corresponding author: C. A. M. Cardoso-Junior, carloscardoso.bio@usp.br

N. A. B. Schumann and C. A. M. Cardoso-Junior contributed equally to this work.

Handling editor: Cedric Alaux (sterile or semi-sterile females) (Michener 2007; Cruz-Landim 2009). Different from bees of the genus Apis and in some stingless bees, in the genus Melipona, the developmental caste fate is not exclusively determined by diet (Campos 1979; Hartfelder and Engels 1998; Faustino et al. 2002). In these bees, diet seems to act as a limiting factor for queen development because morphologically identical larvae that had received a low quantity of food emerge as workers regardless of the genotype (Camargo et al. 1976). For larvae that had received adequate amounts of food, a genetic component is likely involved in 
caste determination since a Mendelian segregation ratio of three workers for each queen has been observed in the offspring of well-fed colonies for many species (Kerr and Nielsen 1966; Kerr et al. 1975; Camargo et al. 1976; Maciel-Silva and Kerr 1991; Jarau et al. 2010). However, the molecular mechanisms related to these developmental routes remain largely unknown.

In Melipona bees, the exogenous treatment with juvenile hormone $(\mathrm{JH})$, a sesquiterpenoid hormone synthetized by the endocrine glandules localized at the retrocerebral complex called corpora allata, is able to promote queen differentiation during the larval stage (Bonetti et al. 1995; Hartfelder and Engels 1998; Judice et al. 2004; Bonetti et al. 2006; Judice et al. 2006; Barchuk et al. 2007; Vieira et al. 2008; Siquieroli et al. 2009). Therefore, JH is responsible for the activation of caste-specific genes. Furthermore, it has been shown that the corpora allata glands of queens are bigger and have larger number of cells than workers of the same age (Kerr et al. 1975; Bonetti et al. 2006). These morphological differences are likely explained by the higher levels of $\mathrm{JH}$ in queens compared to workers (Cardoso et al. 2017a).

In addition to endocrine signals, gene expression can be altered through epigenetic factors acting on chromatin remodeling and consequently altering the accessibility of transcription factors to gene promoters (Allis and Jenuwein 2016). In honeybees (Apis mellifera), epigenetic factors, especially DNA methylation, can mimic the role of the royal jelly influencing the expression of caste-specific genes (Kucharski et al. 2008). The role of epigenetic mechanisms on morphologic and behavioral-associated traits has also been shown in ants (Alvarado et al. 2015; Simola et al. 2016) but remains largely unknown in stingless bees. Recently, epigenetic modifications were associated to caste development in Melipona scutellaris (Cardoso et al. 2017b), suggesting a potential link between epigenetic events and corpora allata activity.

To gain insights into the molecular regulation of phenotypic plasticity of M. scutellaris, we investigated whether the content and dispersion of heterochromatin in the nuclei of corpora allata cells (NCAC) can distinguish queens and workers. Using computationally reconstructed images obtained by confocal microscopy, we have found a different heterochromatin pattern between castes. Our results indicate that the remodeling of the heterochromatin and epigenetic mechanisms may be involved in physiological processes associated with JH synthesis. Thus, this study fills an important gap in the understanding of reproductive division of labor of a non-conventional biological model.

\section{MATERIAL AND METHODS}

\subsection{Bees}

M. scutellaris bees were kept at the Meliponary of the Federal University of Uberlândia (UFU), Uberlândia, MG, Brazil $\left(18^{\circ} 55^{\prime} \mathrm{S} / 45^{\circ} 17^{\prime} \mathrm{W}\right)$. Wax disks containing brood close to emergence were collected from colonies and incubated $\left(31^{\circ} \mathrm{C}-75 \%\right.$ relative humidity) until the newly emerged bees hatched. Three newly emerged queens (NEQ), two newly emerged workers (NEW) and one newly emerged queen produced after JH treatment (NEQ-JH) were used in this study. The nucleus volume, heterochromatin content, and its dispersion were assessed in 65 nuclei of NEW samples, 169 nuclei of NEQ samples, and 26 nuclei of NEQ-JH sample.

\subsection{Treatment with juvenile hormone}

Pre-defecating third larval instar of M. scutellaris $(n=15)$ were collected from the combs and transferred to petri dishes where they were topically treated with a single dose of $5 \mu \mathrm{g}$ of JH III (Sigma-Aldrich, St Louis, MO, USA) diluted in $1 \mu \mathrm{l}$ of acetone (Merck Millipore, Burlington, MA, USA). Control groups ( $n=15)$ were composed by bees treated with acetone (Bonetti et al. 1995). The larvae were kept at $31^{\circ} \mathrm{C}$ with $75 \%$ relative humidity obtained with saturated $\mathrm{KCl}$ solution until adult hatching. Although it is not possible to distinguish morphological differences between queen-destined from workerdestined larvae in Melipona bees, we randomly sampled one individual from a petri dish of $\mathrm{JH}$ treatment in which $100 \%$ of the larvae remained alive and became queens. Thus, we ensured that 
$\mathrm{JH}$ treatment was effective to induce queen-like morphological differences regardless of their genomic background.

\subsection{Preparation of corpora allata of adults for confocal microscopy}

Bees were anesthetized on ice and dissected in physiological solution for insects $(128 \mathrm{mM} \mathrm{NaCl}$, $18 \mathrm{mM} \mathrm{CaCl} 2,1.3 \mathrm{mM} \mathrm{KCl}, 2.3 \mathrm{mM} \mathrm{NaHCO}$ ) under stereoscope (Nikon). Corpora allata glands were stained with YO-PRO-1-iodice, a nuclear fluorescent dye that has been used to observe chromatin domains in fixed and live cells (Flors 2013). Dissected corpora allata glands were permeabilized with TRITON X-100 $(0.1 \%$ $v / v$, Sigma-Aldrich) and sodium citrate $(0.1 \%$ $v / v$ ) and held at room temperature for $1 \mathrm{~h}$. Thereafter, the glands were washed with PBS three times for $5 \mathrm{~min}$ each, then incubated with $1 \mathrm{mM}$ YO-PRO-1-iodice (Thermo Fisher Scientific, Waltham, MA, USA) for $45 \mathrm{~min}$ in a darkroom and finally assembled on a slide with glycerin and enamel-sealed. Images were obtained by confocal microscopy (FluoView 1000, Olympus, Shinjuku, TYO, Japan). Z-stacking was performed with a size of $0.414 \mu \mathrm{m}$ for each optical slice.

\subsection{Nuclei and heterochromatin volume evaluation}

The methodology adopted to analyze and quantify the volume of the nuclei and heterochromatin in M. scutellaris cells is shown in Figure 1 and described elsewhere (de Morais et al. 2018). The following sections describe each step of the methodology.

\subsection{Isotropic resampling, noise reduction, and nuclei segmentation}

All confocal images were gathered into a 3D stack (3D volume), and regions outside the corpora allata were manually removed (Figure 1a). The volume was then resampled to become isotropic, as the original size of the acquisition voxel was not uniform (the slice thickness in the axial dimension ( $z$-axis) resulted in a voxel size in this dimension that is $1.45-20$ times higher than the size in $x$ and $y$ dimensions). Trilinear interpolation was used in the resampling process, resulting in isotropic volumes with voxel size ranging from 0.25 to $0.4 \mu \mathrm{m}$ depending on the sample (Figure 1b) (Schroeder et al. 2006; Costa and Cesar 2009).

Using a filtering process, the noise was reduced in the resampled volume (Figure $1 \mathrm{~b}$ ) when necessary. Two types of noise reduction were used accordingly to the type of the noise: a 3D Gaussian filtering $($ sigma $=1)$ and a median filtering using a $3 \times 3 \times 3$ mask (Schroeder et al. 2006; Costa and Cesar 2009).

A process called segmentation was used to determine which voxels in the volume correspond to the nuclei (Figure 1c). A mixture of different techniques was applied to this step: (i) A simple thresholding (vortex intensity above 20) was used as first draft of the segmentation, which was enough to obtain several nuclei; (ii) an adaptation of 3D Watershed algorithm for those nuclei which were clustered was also used; and (iii) nuclei were split by using opening operation from mathematical morphology, i.e., several erosion operations using a $3 \times 3 \times 3$ cross-shaped mask followed by dilations using the same mask - the dilations were constrained to the voxels of the nuclei detected in the result of the first segmentation using thresholding. After segmentation, the nuclei were labeled using connected component labelling algorithm where nuclei with volume less than $44.7 \mu^{3}$ (empirically obtained) were discarded (Schroeder et al. 2006; Costa and Cesar 2009).

\subsection{Heterochromatin region}

Heterochromatin staining with single-molecule dyes, such as YO-PRO-1-iodice, produces images where the intensity of the voxels is proportional to the chromatin condensation (Simonson et al. 2011; Zessin et al. 2012; Flors 2013). That is the reason why the highest values correspond to the regions of heterochromatin inside the nucleus (Zessin et al. 2012). In order to identify the heterochromatin regions, a new threshold was applied to the resampled volume, but now individually for each nucleus (Figure 1c). The threshold value was chosen as the value corresponding to $40 \%$ above the mean intensity inside the nuclei. 

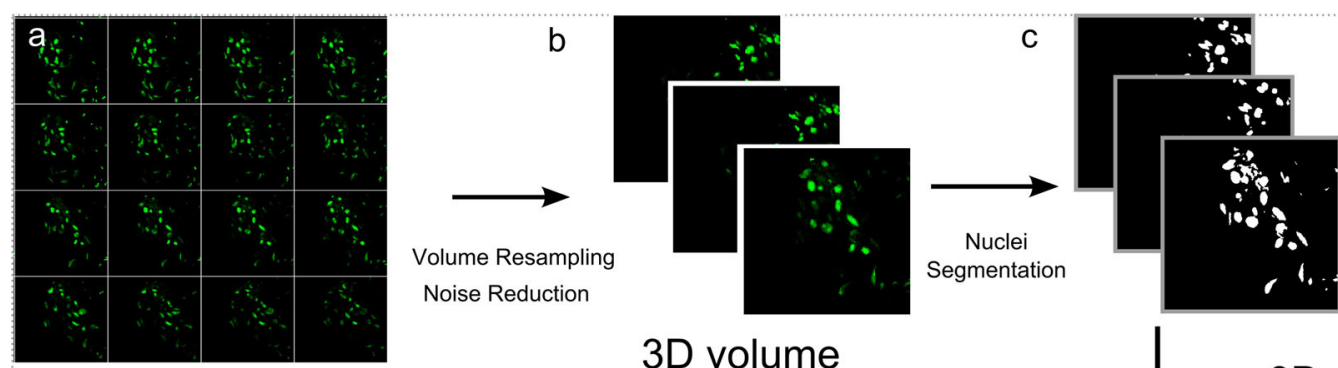

3D volume
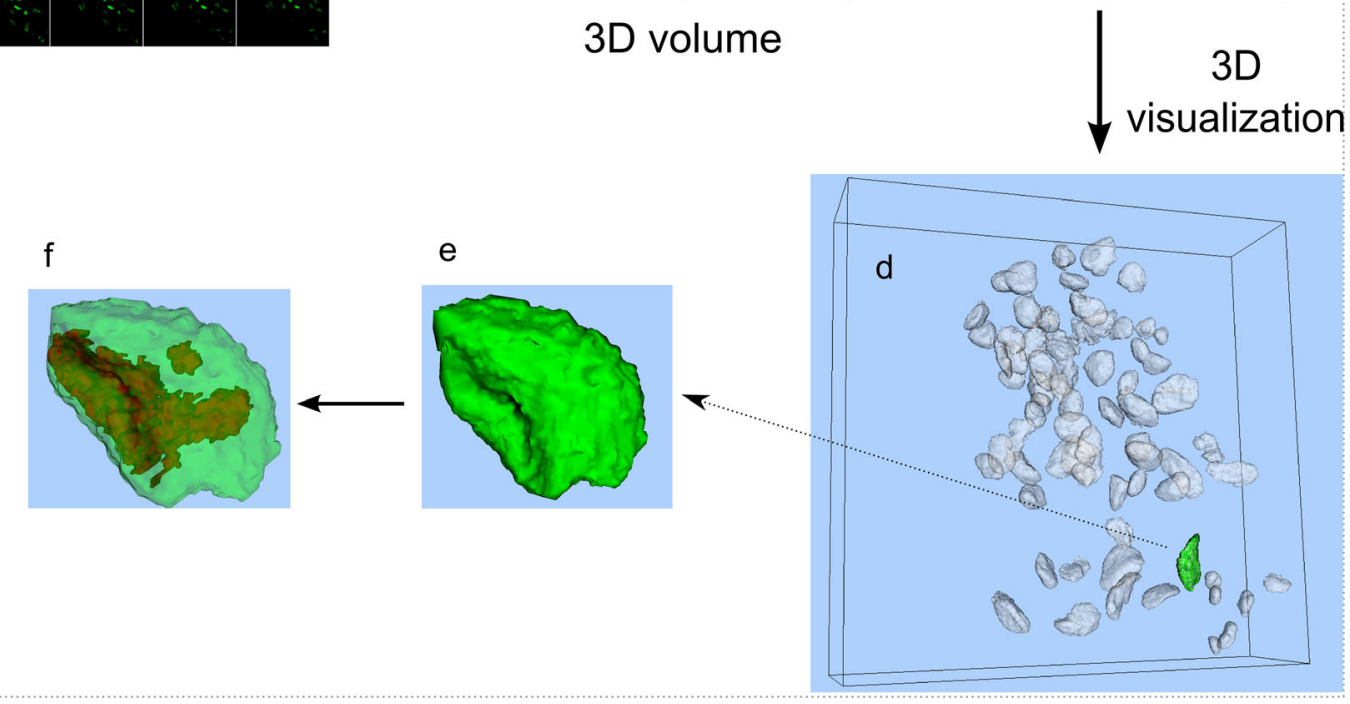

Figure 1 Workflow with the methodology used to analyze and quantify the volume of nuclei and heterochromatin from corpora allata cells in Melipona scutellaris . a Original confocal image series. b 3D volume representation after gathering all images and after the application of algorithms for volume resampling and noise reduction. $\mathbf{c}$ Image segmentation process used for nuclei detection. d 3D reconstruction of all detected nuclei. e An example of a reconstructed nucleus, where it is possible to estimate its volume. $\mathbf{f}$ The same nucleus visualized with a transparent surface (green) and its heterochromatin (brown). Heterochromatin was also detected by using the image segmentation algorithm. At this stage, it is possible to estimate heterochromatin volume.

We used a percentage value above the mean to handle with possible variabilities in the fluorescent intensity of the cells. The same percentage was used in all images. During image acquisition, it may occur differences in the fluorescent intensity between different samples or even between different slices of the same sample, due to fluorescent attenuation along $z$-axis (depth axis). The value of $40 \%$ was chosen empirically, after testing values $20 \%, 40 \%$, and $60 \%$ above the mean.

\subsection{D visualization}

After segmentation, the whole nuclear volume was reconstructed and visualized using marching cube algorithm, generating surfaces composed by triangular meshes surrounding the nuclei and heterochromatin regions. The whole gland was reconstructed (Figure 1d), followed by the visualization of one of the analyzed nucleus (Figure 1e) and its heterochromatin region (Figure 1f, brown region) (Schroeder et al. 2006).

\subsection{Quantitative and qualitative measurements}

In order to estimate the volume of the nuclei $\left(\mu \mathrm{m}^{3}\right)$, the triangle mesh of the surface of the nuclei was used. The same approach was used for heterochromatin volume estimation (Schroeder et al. 2006). We also classified the cells into two groups according to the following 
parameters: type I, "disperse" heterochromatin content was considered with one or more heterochromatin pixels unconnected, usually found in the center and peripheric regions of corpora allata nuclei; and type II, "centralized" heterochromatin content was considered the nuclei with centralized heterochromatin pixels and none splitted pixels. Only the most representative type of heterochromatin dispersion is shown in Figure 2.

\subsection{In silico analysis of regional methylation status}

To gain insights into the epigenetic regulatory mechanisms of genes coding enzymes of JH pathway, the DNA methylation levels were in silico predicted for two genes: farnesoic acid O-methyl transferase (famet) and methyl farnesoate epoxidase $(m f e)$. In silico analysis was carried out using the software Emboss CpGplot (https://www.ebi.ac.uk/Tools/seqstats/emboss_ cpgplot/) and $M$. scutellaris gene sequence available at NCBI website (GenBank accession number: famet, AM493718.1; and $m f e$, KY768854.1). Based on the knowledge that methylated cytosines are hypermutable due to spontaneous deamination, leading to passive demethylation and $\mathrm{CpG}$ depletion over time, the observed to expected ratio (obs/exp) was calculated. This metric determines that regions with an obs/exp. ratio lower than one are predicted as methylated. As $\mathrm{CpG}$ islands are hypomethylated in mammalians and social insects (Fouse et al. 2008; Meissner et al. 2008; Lyko et al. 2010), only regions outside CpG islands were identified as potentially methylated regions (supplementary Figure 1). CpG islands were defined as default by Emboss Cpgplot software $(\mathrm{CG}$ content $=50 \%)$. The obs/exp. approach has been successfully used to access epigenetic information in other social insects (Elango et al. 2009; Kucharski et al. 2015; Glastad et al. 2017).

\subsection{Statistical analysis}

All statistical analyses were performed by using GraphPad Prism software (Version 5.0).
Data were first $\log ^{10}$ transformed to approximate to normal distribution, then tested with D'Agostino-Pearson omnibus test to verify homogeneity distribution of residuals. Differences in the nuclei size and heterochromatin volume between NEW, NEQ, and NEQ-JH were analyzed by ANOVA or Kruskal-Wallis tests, followed by Bonferroni or Dunns correction for multiple comparisons, accordingly to the homogeneity of residuals. Chi-square test was used to assess statistical differences between workers and queens in the number of cells with different heterochromatin patterns. A $p<0.05$ was considered significant.

\section{RESULTS}

Queens and workers differ between their heterochromatin distribution inside NCAC (Figure 2). The majority of NCAC of NEQ show a concentrated heterochromatin at central position in the nucleus, whereas NEW bees present a disperse heterochromatin pattern along NCAC (Figures $2 \mathrm{~g}-\mathrm{i}$ and $3 \mathrm{~d}$ ). We verified that heterochromatin content of NEQ-JH (NEQ raised from JH-treatment) showed a pattern of heterochromatin dispersion similar to NEW (Figure $2 \mathrm{~h}-\mathrm{i}$ ). The quantification of total volume (Figure $3 \mathrm{a}$ ) and heterochromatin content (Figure 3b) demonstrated differences in the total volume between NEQ compared to both NEW and NEQ-JH (ANOVA followed by Bonferroni correction, $F_{(2,257)}=$ $6.36, p<0.05$, Figure $3 a)$. The total heterochromatin content of NCAC from NEQ and NEW individuals was significantly different (ANOVA followed by Bonferroni post hoc test, $F_{(2,257)}=$ 4.566, $p<0.05$, Figure 3b).

In order to verify whether the heterochromatin volume in NEQ samples reflected real differences in caste rather than a proportional increase due to nuclei expansion, we plotted the heterochromatin ratio in relation to total nuclei volume for queens and workers (Figure 3c). With this approach, we did not find any difference in the heterochromatin percentage of NEW and NEQ individuals (MannWhitney test, $U=5417, p>0.05$, Figure 3c), suggesting that the global heterochromatin volume itself is not related to caste-specific phenotypes. 


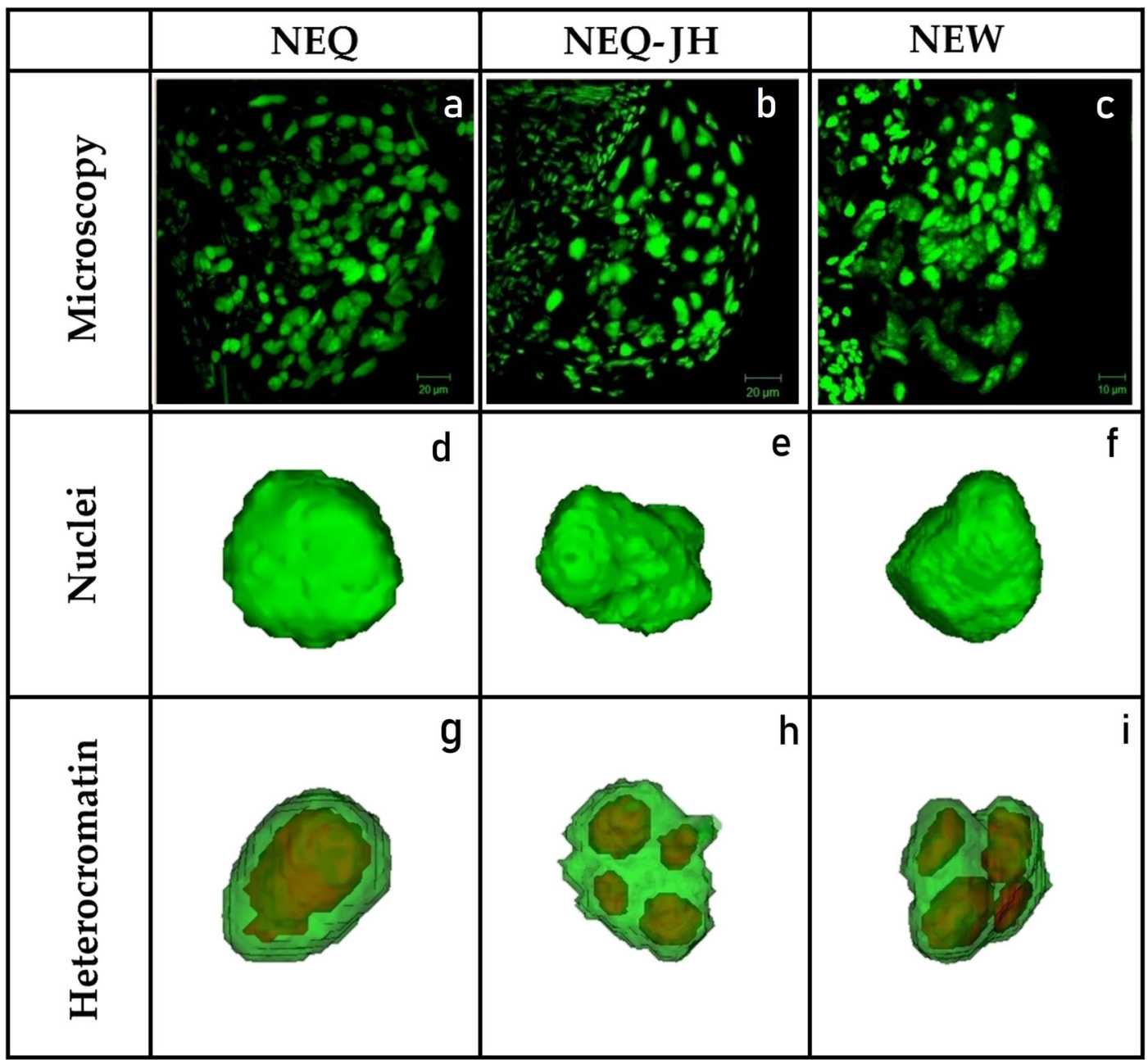

Figure 2 Images of corpora allata cells nuclei of Melipona scutellaris a-c Overall image of the corpora allata gland from a newly emerged workers (NEW), newly emerged queens induced by JH treatment (NEQ-JH) and newly emerged queens (NEQ). $\mathbf{d}-\mathbf{f}$ Computational reconstruction of NEW, NEQ-JH, and NEQ nuclei. $\mathbf{g}-\mathbf{i}$ Dispersion of heterochromatin (brown marks) in NEW, NEQ-JH, and NEQ.

In order to quantitatively evaluate the territorial differences among castes (Figure $3 \mathrm{~d}$ ), the heterochromatin dispersion was grouped into two categories: type I, disperse heterochromatin content; and type II, centralized heterochromatin content. We found a clearly distinct pattern between both castes ( $\chi^{2}$ test, $p<0.0001$, Figure $3 \mathrm{~d}$ ) as previously reported in Figure $2 g$, i. Finally, we used in silico analysis to predict regions that are potentially methylated for two genes (famet and $m f e$ ) which encode critical enzymes involved in the regulation of the $\mathrm{JH}$ biosynthesis. We found two potentially methylated regions flanking a large $\mathrm{CpG}$ island of the famet gene; however, the $m f e$ gene was absent of $\mathrm{CpG}$ islands and presented five potential methylated regions (supplementary Figure 1).

\section{DISCUSSION}

Our data clearly show a distinct territorial dispersion of heterochromatin content and nuclear volume between queens and workers of M. scutellaris stingless bees. These results are in 


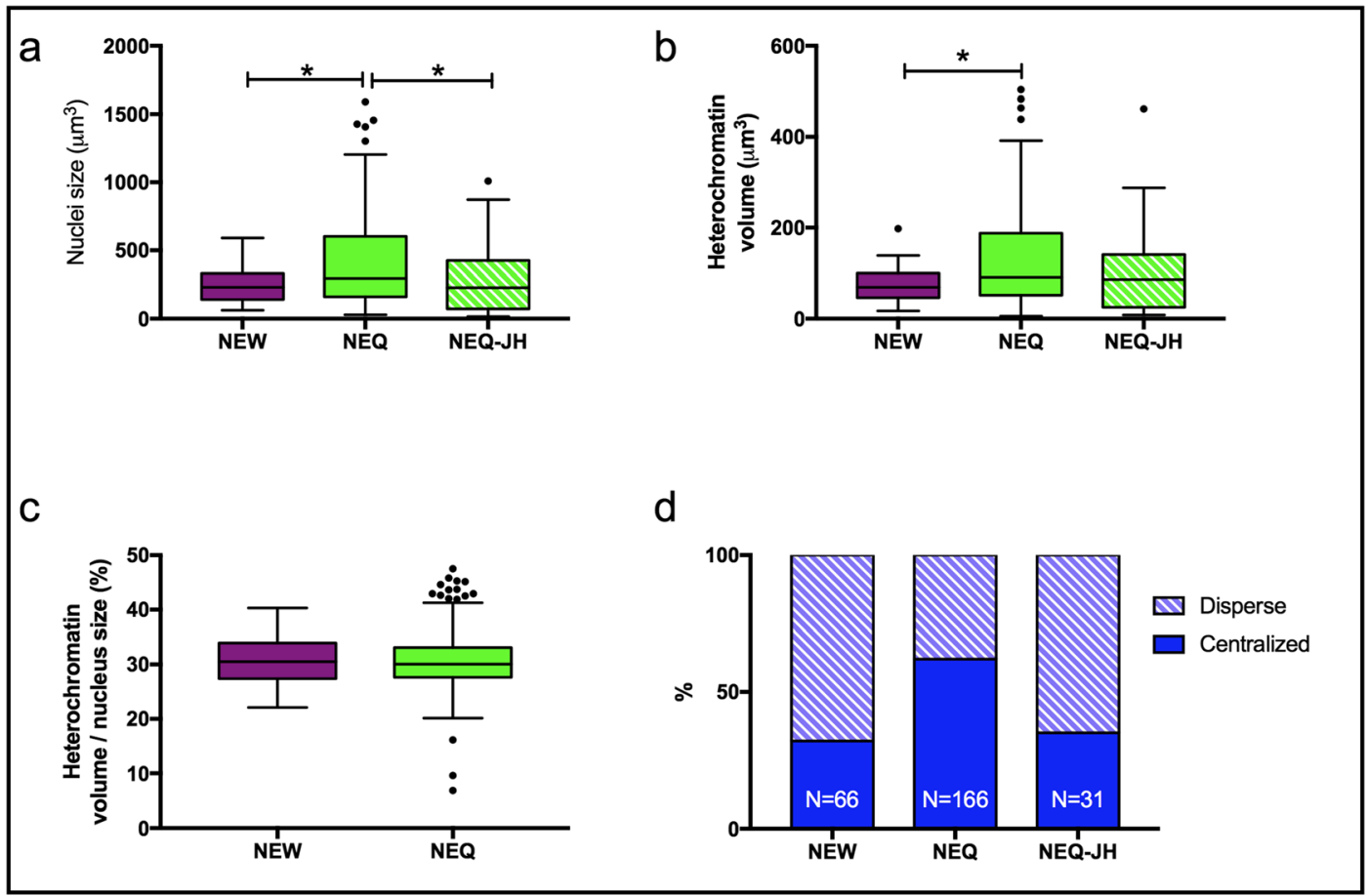

Figure 3 Quantification of heterochromatin and nuclei volume in newly emerged bees of Melipona scutellaris . a Nuclei volume of newly emerged workers (NEW), newly emerged queens (NEQ), and newly emerged queens induced by JH treatment (NEQ-JH). b Total heterochromatin volume of NEW, NEQ-JH, and NEQ. c Percentage of heterochromatin volume in relation to nuclei volume of NEW and NEQ. Number of cells: NEW, $N=65$; NEQ, $N=$ 169; and NEQ-JH, $N=26$. d Classification of heterochromatin dispersion in nuclei of corpora allata cells (sample size is shown inside graphs). The whisker boxes represent the 25 th -75 th percentiles, and dots represent cell samples above or below 1.5 times of intern quantiles; ${ }^{*} p<0.05$.

line with a previous study (Bonetti et al. 2006) suggesting that the three-dimensional reconstruction of NCAC is a suitable approach to evaluate molecular differences between queens and workers of $M$. scutellaris .

Heterochromatin regions are important genomic domains that silence gene expression in eukaryotes (Rea et al. 2000). The heterochromatin is maintained through epigenetic modifications in histone tails and subsequent association of repressor multiprotein complexes (Wang et al. 2014). It has been shown that royal jelly components might facilitate caste switching through modulating histone post-translational modification levels in honeybees (Spannhoff et al. 2011; Wang et al. 2015). The recent mapping of honeybee chromatin structure found several regions with distinct histone post-translational modifications enrichment in the head compartment, which contained corpora allata glands, of both queens and workers larvae (Wojciechowski et al. 2018). Interestingly, differences in the content of histone post-translational modifications were also shown to be castespecific in M. scutellaris (Cardoso et al. 2017b). In addition, differences in heterochromatin dispersion have been previously suggested to regulate genomic silencing in M. scutellaris tissues (Mampumbu and Mello 2006). Therefore, our results suggest that heterochromatin domains and consequently gene silencing are important for the regulation of caste identity in Melipona .

In addition to epigenetic modifications occurring in histone residues, epigenetic modifications in DNA can also affect heterochromatin dispersion and consequently, impact gene expression (Fuks et al. 2000). It is known that DNA 
methylation affects mammalian gene expression reducing the promotor affinity to transcription factors (Bergman and Cedar 2013). In a second stage, methylated $\mathrm{CpG}$ 's sites can recruit repressor complexes to induce heterochromatin regions, ensuring their negative effect on gene expression (Fuks et al. 2000). In contrast to mammalians, DNA methylation in social insects is restricted to gene-body regions and regulates gene expression and splicing events (Lyko et al. 2010; Li-Byarlay et al. 2013; Alvarado et al. 2015). Interestingly, dynamic fluctuations of global levels of DNA methylation were found throughout critical developmental steps of caste dimorphism in Melipona bees (Cardoso et al. 2017b) and also in other social insects, as honeybees and fire ant Solenopsis invicta (Lyko et al. 2010; Foret et al. 2012; Kay et al. 2016). Together, these findings suggest a potential cross-talk between DNA methylation and heterochromatin regions regulating the phenotypic plasticity in stingless bee.

$\mathrm{JH}$ has been used to explain the control of endocrine mechanisms associated with metamorphosis, reproduction, development of the gonads, determination of caste, behavior, polyphenism, and physiology in a large range of insects (Bonetti et al. 1995; Hartfelder and Engels 1998; Bonetti et al. 2006; Barchuk et al. 2007). Interestingly, the larval methylomes of $A$. mellifera show differences between queens and workers in genes related to $\mathrm{JH}$ synthesis pathway (Foret et al. 2012). It is attempting to speculate that $m f e$, a gene from $\mathrm{JH}$ pathway, is also regulated by epigenetic mechanisms. Some evidences support this potential regulatory link between epigenetic mechanisms and $\mathrm{JH}$ pathway in the context of caste differentiation: (i) $m f e$ encodes the last enzyme of JH pathway, and its expression is restricted to corpora allata cells (Cardoso et al. 2017a); (ii) its expression is higher expressed in queens compared to workers (Cardoso et al. 2017a); (iii) its transcriptional profile correlates with corpora allata activity (Cardoso et al. 2017a) which has a different heterochromatin pattern between castes (this study); and (iv) potentially methylated regions were found in $m f e$ genomic sequence (this study). Corroborating this hypothesis, we also found potential methylated regions in the famet gene, another gene from $\mathrm{JH}$ pathway that is differentially expressed and spliced in both castes of M. scutellaris (Vieira et al. 2008).
Forthcoming studies must uncover the epigenetic control of JH-related enzymes in order to enlighten the caste determination system in Melipona bees.

JH treatment had no direct effect on chromatin remodeling despite differences in adult phenotype. This curious result is in line with previous published data (Judice et al. 2006) which identified differences in the expression of caste-specific genes in NEQ and NEQ-JH of $M$. quadrifasciata. In addition, DNA methylation and $\mathrm{JH}$ hormone seem to be independent regulatory modules in young workers in honeybees (Cardoso et al. 2018). Although we focused on a developmental stage where the castes were already determined, this result suggests that $\mathrm{JH}$ is a downstream component of caste differentiation, rather than affecting heterochromatin dispersion on NCAC. Whether heterochromatin patterns of NCAC affect JH biosynthesis remains to be determined.

Our results shed light on developmental processes associated to caste differentiation of Melipona bees. More specifically, they indicate that gene silencing through heterochromatin patterns is a molecular mark of caste identity in Melipona and therefore that epigenetic mechanisms may be associated to physiological signals to regulate social traits in stingless bees.

\section{ACKNOWLEDGEMENTS}

The authors thank Dr. Klaus Hartfelder from USPFMRP for providing critical comments that helped to improve this manuscript and Dr. Carlos Henrique de Carvalho from UFU for his efforts to obtain the biological material used in this work.

\section{AUTHORS' CONTRIBUTION}

BAM, UVC, and BME conceived and designed experiments. BNA, BLM, and FMB performed experiments. CJCAM, SHE, and TBAN analyzed the data. CJCAM, AGR, TBAN, and BAM wrote the paper. BAM and TBAN supervised the work. All authors read and approved the final manuscript. 


\section{FUNDING INFORMATION}

This work was supported by grants from "Fundação de Amparo à Pesquisa do Estado de São Paulo" (2016/15881-0 and 2017/09269-3 to CJCAM), "Fundação de Amparo à Pesquisa do Estado de Minas Gerais" (APQ-01919-14 and APQ-01579-09 to BAM and APQ-03437-15 to TBAN).

\section{COMPLIANCE WITH ETHICAL STANDARDS}

Conflict of interest The authors declare that they have no conflict of interest.

\section{REFERENCES}

Allis C.D., T. Jenuwein. (2016) The molecular hallmarks of epigenetic control. Nat. Rev. Genet. 17 (8), 487-500.

Alvarado S., R. Rajakumar, E. Abouheif, M. Szyf. (2015) Epigenetic variation in the Egfr gene generates quantitative variation in a complex trait in ants. Nat. Commun. 6, 6513.

Barchuk A.R., A.S. Cristino, R. Kucharski, L.F. Costa, Z.L. Simoes, et al. (2007) Molecular determinants of caste differentiation in the highly eusocial honeybee Apis mellifera. BMC Dev. Biol. 7, 70.

Bergman Y., H. Cedar. (2013) DNA methylation dynamics in health and disease. Nat. Struct. Mol. Biol. 20(3), 274-281.

Bonetti A.M., W.E. Kerr, S.H. Matusita. (1995) Effects of juvenile hormones I, II and III, in single and fractionated dosage in Melipona bees. Rev. Bras. Biol. 55 Suppl 1, 113-120.

Bonetti A.M., A.C.S. Siquieroli, F.A. Santana, C.U. Vieira, M. Bezerra, et al. (2006) Effect of juvenile hormone III on the ultrastructure of the corpora allata in Melipona quadrifasciata (Hymenoptera, Apidae, Meliponini). Braz. J. Morphol. Sci. 23, 393-400.

Camargo, C.A., Almeida, M.G., Parra, M.G.N., Kerr, W.E. (1976) Genetics of sex determination in bees. IX. Frequencies of queens and workers from larvae under controlled conditions (Hymenoptera: Apoidea). J. Kansas Entomol. Soc. 49, 120-125.

Campos L.A.O. (1979) Determinação do sexo nas abelhas XIII. Determinação das castas em Partamona cupira (Hymenoptera, Apidae). Papel do Hormônio Juvenil. Cien. Cult. 31, 65-70.

Cardoso C.A.M., R.P. Silva, N.A. Borges, W.J. de Carvalho, S.L. Walter, et al. (2017a) Methyl farnesoate epoxidase $(m f e)$ gene expression and juvenile hormone titers in the life cycle of a highly eusocial stingless bee, Melipona scutellaris. J. Insect Physiol. 101, 185-194.

Cardoso C.A.M., P.T. Fujimura, C.D. Santos, N.A. Borges, C. Ueira-Vieira, et al. (2017b) Epigenetic modifications and their relation to caste and sex determination and adult division of labor in the stingless bee Melipona scutellaris . Genet. Mol. Biol. 40 (1), 61-68.

Cardoso C.A.M., K.R. Guidugli-Lazzarini, K. Hartfelder. (2018) DNA methylation affects the lifespan of honey bee (Apis mellifera L.) workers - Evidence for a regulatory module that involves vitellogenin expression but is independent of juvenile hormone function. Insect Biochem. Mol. Biol. 92 , 21-29.

Costa L., R. Cesar. (2009) Shape Classification and Analysis: Theory and Practice. CRC Press. Boca Raton, USA.

Cruz-Landim C. (2009) Abelhas: Morfologia e Função de Sistemas. UNESP ed. São Paulo, Brazil.

de Morais C.R., B.A.N. Travencolo, S.M. Carvalho, M.E. Beletti, V.S. Vieira Santos, et al. (2018) Ecotoxicological effects of the insecticide fipronil in Brazilian native stingless bees Melipona scutellaris (Apidae: Meliponini). Chemosphere 206, 632-642.

Elango N., B.G. Hunt, M.A. Goodisman, S.V. Yi. (2009) DNA methylation is widespread and associated with differential gene expression in castes of the honeybee, Apis mellifera. Proc. Natl. Acad. Sci. USA 106 (27), 11206-11211.

Faustino C., E. Silva Matos, S. Mateus, R. Zucchi. (2002) First record of emergency queen rearing in stingless bees (Hymenoptera, Apinae, Meliponini). Insectes Sociaux 49 (2), 111-113.

Flors C. (2013) Super-resolution fluorescence imaging of directly labelled DNA: from microscopy standards to living cells. J Microsc. 251 (1), 1-4.

Foret S., R. Kucharski, M. Pellegrini, S. Feng, S.E. Jacobsen, et al. (2012) DNA methylation dynamics, metabolic fluxes, gene splicing, and alternative phenotypes in honey bees. Proc. Natl. Acad. Sci. USA. 109 (13), 4968-4973.

Fouse S.D., Y. Shen, M. Pellegrini, S. Cole, A. Meissner, et al. (2008) Promoter CpG methylation contributes to ES cell gene regulation in parallel with Oct $4 / \mathrm{Nanog}$, PcG complex, and histone $\mathrm{H} 3 \mathrm{~K} 4 / \mathrm{K} 27$ trimethylation. Cell Stem Cell 2 (2), 160-169.

Fuks F., W.A. Burgers, A. Brehm, L. Hughes-Davies, T. Kouzarides. (2000) DNA methyltransferase Dnmt1 associates with histone deacetylase activity. Nat. Genet. $24(1), 88-91$.

Glastad K.M., S.V. Arsenault, K.L. Vertacnik, S.M. Geib, S. Kay, et al. (2017) Variation in DNA Methylation Is Not Consistently Reflected by Sociality in Hymenoptera. Genome Biol. Evol. 9 (6), 1687-1698.

Hartfelder K., W. Engels. (1998) Social insect polymorphism: hormonal regulation of plasticity in development and reproduction in the honeybee. Curr. Top. Dev. Biol. 40, 45-77. 
Jarau, S., van Veen, J.W., Twele, R., Reichle, C., Gonzales, E.H., Aguilar, I. Francke, W., Ayasse, M. (2010) Workers make the queens in Melipona bees: identification of geraniol as a caste determining compound from labial glands of nurse bees. J. Chem. Ecol. 36, $565-569$.

Judice C.C., K. Hartfelder, G.A. Pereira. (2004) Castespecific gene expression profile in the stingless bee Melipona quadrifasciata - Are these common patterns in highly eusocial bees? Insectes Sociaux 51, 352358.

Judice C.C., M.F. Carazzole, F. Festa, M.C. Sogayar, K. Hartfelder, et al. (2006) Gene expression profiles underlying alternative caste phenotypes in a highly eusocial bee, Melipona quadrifasciata. Insect Mol. Biol. $15(1), 33-44$.

Kay S., D. Skowronski, B.G. Hunt. (2016) Developmental DNA methyltransferase expression in the fire ant Solenopsis invicta. Insect Sci. 25(1), 57-65.

Kerr W.E., Nielsen, R.A. (1966) Evidences that genetically determined Melipona queens can become workers. Genetics, 54, 859-866.

Kerr W.E., Y. Akahira, C.A. Camargo. (1975) Sex determination in bees. IV. Genetic control of juvenile hormone production in Melipona quadrifasciata (Apidae). Genetics 81 (4), 749-756.

Kucharski R., J. Maleszka, S. Foret, R. Maleszka. (2008) Nutritional control of reproductive status in honeybees via DNA methylation. Science 319 (5871), 18271830.

Kucharski R., S. Foret, R. Maleszka. (2015) EGFR gene methylation is not involved in Royalactin controlled phenotypic polymorphism in honey bees. Sci. Rep. 5, 14070.

Li-Byarlay H., Y. Li, H. Stroud, S. Feng, T.C. Newman, et al. (2013) RNA interference knockdown of DNA methyl-transferase 3 affects gene alternative splicing in the honey bee. Proc. Natl. Acad. Sci. USA. 110 (31), $12750-12755$.

Lyko F., S. Foret, R. Kucharski, S. Wolf, C. Falckenhayn, et al. (2010) The honey bee epigenomes: differential methylation of brain DNA in queens and workers. PLoS Biol. 8(11), e1000506.

Maciel-Silva, V.L., Kerr, W.E. (1991) Sex determination in bees. XXVII. Castes obtained from larvae fed homogenized food in Melipona compressipes (Hymenoptera, Apidae). Apidologie, 22, 15-19.

Mampumbu A.R., M.L. Mello. (2006) DNA methylation in stingless bees with low and high heterochromatin contents as assessed by restriction enzyme digestion and image analysis. Cytometry A 69 (9), 986-991.

Meissner A., T.S. Mikkelsen, H. Gu, M. Wernig, J. Hanna, et al. (2008) Genome-scale DNA methylation maps of pluripotent and differentiated cells. Nature 454 (7205), 766-770.
Michener C.D. (2007) The bees of the world. Johns Hopkins University Press, Baltimore.

Rea S., F. Eisenhaber, D. O'Carroll, B.D. Strahl, Z.W. Sun, et al. (2000) Regulation of chromatin structure by sitespecific histone H3 methyltransferases. Nature 406 (6796), 593-599.

Schroeder W.J., Martin K.M, Lorensen B. (2006) The visualization toolkit $\left(4^{\text {th }}\right.$ ed.). Kitware, New York

Simola D.F., R.J. Graham, C.M. Brady, B.L. Enzmann, C. Desplan, et al. (2016) Epigenetic (re)programming of caste-specific behavior in the ant Camponotus floridanus. Science 351 (6268), aac6633.

Simonson P.D., E. Rothenberg, P.R. Selvin. (2011) Singlemolecule-based super-resolution images in the presence of multiple fluorophores. Nano Lett. 11 (11), 5090-5096.

Siquieroli A.C., C.U. Vieira, G.A. Carvalho-Zilse, L.R. Goulart, W.E. Kerr, et al. (2009) Analysis of the intercaste transcriptional profile of Melipona scutellaris Latreille, 1811 (Hymenoptera, Apidae, Meliponini) by mRNA differential display. Biol. Res. 42 (1), 107110 .

Spannhoff A., Y.K. Kim, N.J. Raynal, V. Gharibyan, M.B. $\mathrm{Su}$, et al. (2011) Histone deacetylase inhibitor activity in royal jelly might facilitate caste switching in bees. EMBO Rep. 12 (3), 238-243.

Vieira C.U., A.M. Bonetti, Z.L. Simoes, A.Q. Maranhao, C.S. Costa, et al. (2008) Farnesoic acid O-methyl transferase (FAMeT) isoforms: conserved traits and gene expression patterns related to caste differentiation in the stingless bee, Melipona scutellaris . Arch. Insect. Biochem. Physiol. 67 (2), 97-106.

Wang J., S.T. Lawry, A.L. Cohen, S. Jia. (2014) Chromosome boundary elements and regulation of heterochromatin spreading. Cell. Mol. Life Sci. 71 (24), 48414852.

Wang W.X., L. Tian, Q. Huang, X.B. Wu, Z.J. Zeng. (2015) Effects of 10-Hydroxy-2-decenoic acid on the development of honey bee (Apis mellifera) larvae. J. Apic. Res. 53 (1), 171-176.

Wojciechowski M., R. Lowe, J. Maleszka, D. Conn, R. Maleszka, et al. (2018) Phenotypically distinct female castes in honey bees are defined by alternative chromatin states during larval development. Genome Res. $28(10), 1532-1542$.

Zessin P.J., K. Finan, M. Heilemann. (2012) Superresolution fluorescence imaging of chromosomal DNA. J. Struct. Biol. 177 (2), 344-348.

Publisher's note Springer Nature remains neutral with regard to jurisdictional claims in published maps and institutional affiliations. 